\title{
The Relationship Between Blood Interleukin-10 and Cardiovascular Mortality and All-Cause Mortality After Kidney Transplantation
}

This article was published in the following Dove Press journal: Risk Management and Healthcare Policy

\section{Chen Gao \\ Fenghua Peng \\ Xubiao Xie \\ Longkai Peng}

Department of Kidney Transplantation, The Second Xiangya Hospital of Central South University, Changsha, Hunan, 4I00II, People's Republic of China
Correspondence: Longkai Peng Department of Kidney Transplantation, The Second Xiangya Hospital of Central South University, Changsha, Hunan, 4I00II, People's Republic of China Email penglongkai@csu.edu.cn
Background: Circulating interleukin (IL)-10 is associated with adverse cardiovascular events in chronic kidney disease (CKD). Whether IL-10 predicts cardiovascular and allcause mortality after kidney transplantation (KT) is unknown.

Methods: The association between plasma IL-10 and cardiovascular and all-cause mortality was analyzed in a prospective cohort, which included 418 stable kidney transplant recipients, at a median of 3.6 (range $=1.2-8.4$ ) years after transplantation. Multivariate Cox regression models were performed to adjusting related confounding factors.

Results: Median level of IL-10 in KT patient was $22.3 \mathrm{pg} / \mathrm{mL}$. Multivariate Cox regression analysis revealed that serum levels IL-10 were significantly and independently associated with cardiovascular mortality after adjusting for age, gender, BMI, current smoker, current drinker, cause of kidney disease, systolic and diastolic BP, laboratory indexes and medication $(\mathrm{HR}=1.26,95 \%$ CI $1.19-2.08, P$-trend $<0.001)$. The multivariate Cox analysis also suggested that serum levels IL-10 were independently associated with all-cause mortality $(\mathrm{HR}=1.25$, 95\% CI 1.11-1.8, $P$-trend $=0.023$ ) after controlling these same related confounding factors. Sensitivity and stratified analysis showed that the significant association can be affected by history of acute rejection.

Conclusion: Plasma IL-10 is independently and significant associated with cardiovascular and all-cause mortality after kidney transplantation. The significant association is independent of cardiovascular risk factors and other related confounding factors.

Keywords: IL-10, kidney transplantation, cardiovascular mortality, all-cause mortality, Cox regression, prognosis

\section{Introduction}

Kidney transplantation (KT) is the ideal treatment for patients with end-stage renal disease (ESRD). ${ }^{1}$ In the past few decades, the short-term and long-term prognosis of KT recipients has dramatically improved due to improved surgical techniques, prevention of infections and better immunosuppressive therapies. However, compared with the general population, the risk of cardiovascular mortality is markedly elevated in KT recipients. $^{2-4}$

Interleukin (IL)-10 is an immunoregulatory cytokine that plays an important role in regulating proinflammatory and anti-inflammatory responses. ${ }^{5}$ In addition to demonstrating anti-inflammatory effects in several experimental studies, IL-10 has also been shown to possess antiatherosclerotic and antithrombotic properties. ${ }^{6}$ Interestingly, some clinical studies have found that higher serum levels of IL-10 
were associated with improved clinical outcomes, including cardiovascular morbidity and mortality. ${ }^{7}$ However, another study showed the opposite results. ${ }^{8}$ A potential explanation for this inconsistency is that the kinetics of IL-10 are different in patients with CKD. ${ }^{9,10}$ Previous evidence has also suggested large interindividual differences in the serum levels of IL-10 in patients who have undergone hemodialysis. A chronic inflammatory state is common in KT recipients with impaired renal function and in patients with chronic kidney disease (CKD). ${ }^{11-14}$ In CKD patients, existing evidence has suggested that circulating levels of IL-10, IL-6, C-reactive protein and other inflammatory cytokines are important risk factors for predicting the development of cardiovascular diseases (CVDs) and all-cause mortality. ${ }^{15-18}$ Whether IL-10 can be used to predict cardiovascular mortality in KT recipients is unknown.

To date, few studies have investigated whether serum IL-10 is associated with cardiovascular and all-cause mortality in KT patients. In view of the past evidence, we aimed to evaluate whether serum IL-10 is associated with the development of cardiovascular and all-cause mortality in KT patients.

\section{Materials and Methods}

KT recipients who had graft functioning for at least 1 year and visited our outpatient service between January 2010 and December 2012 were included in this prospective cohort study. Serum samples collected from 418 patients were available. These patients were enrolled at after transplantation and then were followed up. Patients diagnosed with signs of infection, cancer or other serious diseases were not included. The Ethics Committee of The Second Xiangya Hospital of Central South University approved the study protocol, following the principles of the Declaration of Helsinki. All KT patients provided written informed consent.

\section{Follow-Up}

KT patients were followed up by telephone and/or by reviewing their medical records 4 times a year until the occurrence of an endpoint event. The endpoints of the study were cardiovascular mortality and all-cause mortality. Adequate collection of endpoint events was ensured by our continuous and reliable surveillance system. If the current status of the KT patients was unknown, their referring nephrologists and/or general practitioners were contacted and asked to identify the patient's condition. For the purpose of this study, cardiovascular death was defined as death caused by myocardial infarction, stroke, heart failure or sudden cardiac death.

\section{Laboratory Measurements}

All blood samples were obtained from KT patients in the morning after 12 hours of fasting for the measurement of serum basal insulin, serum fasting plasma glucose, serum triglyceride, total cholesterol, high-density lipoprotein (HDL), low-density lipoprotein (LDL) and serum albumin. C-reactive protein, IL-10 and IL-6 were tested at the same time. Homeostasis model assessment-insulin (HOMA-IR) was calculated to obtain an insulin resistance score. ${ }^{19} \mathrm{C}$-reactive protein was tested using a photometric method. Serum levels of IL-10 were measured by a commercially available sandwich enzyme immunoassay kit (Human IL-10 Platinum ELISA Kit; eBioscience, Bender MedSystems, Vienna, Austria). The overall intraassay and interassay coefficients of variation were 3.2\% and $5.6 \%$, respectively. Serum levels of IL-6 were tested using human IL-6 ELISA kits (Quantikine HS Human IL6 Immunoassay; R\&D Systems, Minneapolis, MN) with an interassay coefficient of variation of $7.8 \%$ and a sensitivity of $0.16 \mathrm{pg} / \mathrm{mL}$. The eGFR was calculated by using the CKD Epidemiology Collaboration equation. $^{20}$

\section{Statistical Analyses}

All of the data were analyzed by using SPSS 26.0. $P \leq$ 0.05 was considered to be statistically significant. Variable distribution was tested with probability plots and histograms. Variables that are normally distributed are presented as the mean $\pm \mathrm{SD}$, and skewed variables are presented as the median (interquartile range). Baseline characteristics were compared between two subgroups (patients with IL-10 $<22.3 \mathrm{pg} / \mathrm{mL}$ and patients with IL$10 \geq 22.3 \mathrm{pg} / \mathrm{mL}$ ) according to the median value of IL-10 using the Kruskal-Wallis test, one-way ANOVA and chisquare tests as appropriate. Skewed data were natural logtransformed for correlation and Cox regression analysis. In the multivariate analysis, an adjustment for the confounding factors age and sex was made in Model 1. Adjustments for the confounding factors age, sex, body mass index (BMI), current smoking status, current drinking status, cause of kidney disease, systolic and diastolic blood pressure (BP) and laboratory indexes were performed in Model 2. In Model 3, adjustments for age, sex, BMI, current smoker, current drinker, cause of kidney disease, systolic 
and diastolic BP, laboratory indexes and medication were made.

Serum levels of IL-10 at baseline were categorized by quartiles (quartile 1: $\leq 25$ th percentile, quartile $2: 25$ th to 50th percentile, quartile $3: 50$ th to 75 th percentile, quartile 4: $\geq 75$ th percentile). Cox regression analysis was performed to identify the independent predictors of cardiovascular mortality and all-cause mortality in KT patients. To further evaluate the independent association, we excluded the effect of "anti-inflammatory medication" in the sensitivity analysis. Finally, we analyzed the association between IL-10 levels at baseline and cardiovascular mortality and all-cause mortality during the follow-up by stratified analysis. CV event-free curves were constructed by the Kaplan-Meier method, and the Log rank test was performed.

\section{Results}

\section{Clinical Characteristics of the KT Patients at Baseline}

The clinical features of the KT patients are described in Table 1. All included patients with KT were divided into two groups according to their median value of IL-10 (22.3 $\mathrm{pg} / \mathrm{mL}$ ). KT patients with higher levels of serum IL-10 $(\geq 22.3 \mathrm{pg} / \mathrm{mL}$ ) had higher cardiovascular mortality and allcause mortality than those who had lower levels of serum IL-10 $(<22.3 \mathrm{pg} / \mathrm{mL})$, which initially suggested that elevated IL-10 levels were directly associated with increased cardiovascular and all-cause mortality. KT patients with high serum IL-10 levels had a higher BMI, a longer dialysis duration, elevated systolic and diastolic BP, and tended to be current smokers and drinkers. Information about their laboratory indexes and medications is described in Table 1.

\section{Elevated Serum Levels of IL-10 Were Independently Associated with an Increased Risk of Cardiovascular Mortality and All-Cause Mortality in 4I9 Patients with KT}

All included patients $(\mathrm{N}=419)$ were prospectively followed up during a median of 3.6 (range=1.2-8.4) years after transplantation. Cardiovascular mortality occurred in 25 KT patients, including myocardial infarction $(\mathrm{N}=11)$, stroke $(\mathrm{N}=7)$, heart failure $(\mathrm{N}=4)$ and sudden cardiac death $(\mathrm{N}=3)$, during the follow-up period. Kaplan-Meier analysis suggested that KT patients with serum IL-10 levels above the median $(22.3 \mathrm{pg} / \mathrm{mL})$ had a higher rate of cardiovascular mortality (Log rank test, $P=0.006$, Figure 1) and all-cause mortality than KT patients with serum IL-10 levels below the median value (Log rank test, $P=0.037$, Figure 2).

To further evaluate the association of serum IL-10 levels with cardiovascular mortality and all-cause mortality in KT patients, a multivariate Cox proportional hazard regression model was performed. Cox analysis revealed that elevated serum IL-10 levels were independently and significantly associated with an increased risk of cardiovascular mortality $(\mathrm{HR}=1.26,95 \%$ CI $1.19-2.08$, $P$-trend $<0.001$, Table 2) and all-cause mortality $(\mathrm{HR}=1.25,95 \%$ CI 1.11-1.82, $P$-trend $=0.023$, Table 3$)$, respectively, after adjustments for age, sex, BMI, current smoker, current drinker, cause of kidney disease, systolic and diastolic BP, laboratory indexes (creatinine, albumin, calcium, phosphate, IL-6, C-reactive protein, estimated GFR, NT-proBNP, total cholesterol, triglycerides, HDL cholesterol, LDL cholesterol and glucose) and medication were made in Model 3.

To further clarify the associations between serum IL-10 levels and cardiovascular mortality and all-cause mortality in patients with $\mathrm{KT}$, an additional sensitivity analysis was performed by adding "dialysis duration" as a covariate. Our results still showed that elevated IL-10 levels were significantly associated with a higher risk of cardiovascular mortality $(\mathrm{HR}=1.24,95 \% \mathrm{CI} 1.12-1.98, P$-trend $<0.001$, Table 4) and all-cause mortality $(\mathrm{HR}=1.22,95 \%$ CI 1.09 1.87, $P$-trend $=0.025$, Table 5) after adjusting for these confounding factors.

\section{Elevated Serum Levels of IL-10 Were Independently Associated with an Increased Risk of Cardiovascular Mortality and All-Cause Mortality by Stratified Analysis in 419 KT Patients}

An independent association with IL-10 may be affected by a history of acute rejection. Thus, stratified analysis by adding "history of acute rejection" as a covariate was performed (Table 6). Our results suggested that serum IL-10 levels were independently associated with an increased risk of cardiovascular mortality $(\mathrm{HR}=1.40$, 95\% CI 1.16-2.85, $P$-trend $=0.026)$ and all-cause mortality $(\mathrm{HR}=1.34,95 \%$ CI $1.13-2.67, P$-trend $=0.031)$ in $\mathrm{KT}$ patients with a history of acute rejection but not in those 
Table I Clinical Characteristics of KT Patients at Baseline (N=419)

\begin{tabular}{|c|c|c|c|}
\hline Characteristics & IL- $10<22.3 \mathrm{pg} / \mathrm{mL}(\mathrm{N}=209)$ & IL-I $0 \geq 22.3 \mathrm{pg} / \mathrm{mL}(\mathrm{N}=2 \mid 0)$ & $P$ value \\
\hline Age $(Y)$ & $56.4 \pm 9.2$ & $57.2 \pm 9.5$ & 0.079 \\
\hline Gender (male), n (\%) & $117(56.0)$ & $102(48.6)$ & 0.106 \\
\hline Cardiovascular mortality, n (\%) & $8(3.8)$ & $17(8.1)$ & $<0.001$ \\
\hline All-cause mortality, n (\%) & $12(5.7)$ & $26(12.4)$ & $<0.001$ \\
\hline Current smoker, n (\%) & $64(30.6)$ & $72(34.3)$ & 0.014 \\
\hline Current drinker, n (\%) & $82(39.2)$ & $103(49.0)$ & $<0.001$ \\
\hline BMI $\left(\mathrm{kg} / \mathrm{m}^{2}\right)$ & $25 \pm 4.8$ & $27 \pm 5.4$ & 0.002 \\
\hline Cause of kidney disease & & & 0.531 \\
\hline Primary Glomerulopathy, n (\%) & $104(49.8)$ & $99(47.1)$ & \\
\hline Hypertension, n (\%) & $31(14.8)$ & $28(13.3)$ & \\
\hline Diabetes, n (\%) & $12(5.7)$ & I5 (7.I) & \\
\hline Other/unknown, n (\%) & $62(29.7)$ & $68(32.4)$ & \\
\hline Dialysis duration (months) & $24.6 \pm 3.1$ & $29.4 \pm 3.7$ & $<0.001$ \\
\hline History of acute rejection, $\mathrm{n}(\%)$ & $71(34.3)$ & $74(35.2)$ & 0.637 \\
\hline Systolic BP (mmHg) & $154 \pm 25.6$ & $166 \pm 29.4$ & $<0.001$ \\
\hline Diastolic BP (mmHg) & $91 \pm 8.7$ & $95 \pm 10.6$ & $<0.001$ \\
\hline \multicolumn{4}{|l|}{ Laboratory indexes } \\
\hline Creatinine (mg/dl) & $1.47(1.13-1.73)$ & $1.98(1.42-2.53)$ & $<0.001$ \\
\hline Albumin $(\mathrm{g} / \mathrm{dl})$ & $4.12 \pm 0.32$ & $3.87 \pm 0.29$ & $<0.001$ \\
\hline Calcium (mg/dl) & $9.2 \pm 0.5$ & $9.5 \pm 0.6$ & 0.003 \\
\hline Phosphate (mg/dl) & $4.3 \pm 0.3$ & $6.3 \pm 0.4$ & $<0.001$ \\
\hline IL-6 (pg/mL) & $5.2(1.0-6.6)$ & 12. (1.3-27.4) & $<0.001$ \\
\hline IL-I0 (pg/mL) & $12.6(4.8-20.3)$ & $45.2(24 .|-6| .5)$ & $<0.001$ \\
\hline C-reactive protein $(\mathrm{mg} / \mathrm{L})$ & $8.2(3.5-16.5)$ & $23.6(6.3-37.5)$ & $<0.001$ \\
\hline Estimated GFR (mL/min per $1.73 \mathrm{~m} 2)$ & $54.3 \pm 14.8$ & $38.6 \pm 12.1$ & $<0.001$ \\
\hline NT-proBNP (pg/mL) & $286.4(96.7-505.8)$ & $489.5(247.8-1544.9)$ & $<0.001$ \\
\hline Total cholesterol (mg/dl) & $215.6 \pm 3 \mid .4$ & $204.3 \pm 34.5$ & 0.191 \\
\hline Triglycerides (mg/dl) & $14 \mid .9 \pm 14.6$ & $142 \pm 13.2$ & 0.357 \\
\hline HDL cholesterol (mmol/L) & $44.9 \pm 12.3$ & $40.4 \pm 11.7$ & $<0.001$ \\
\hline LDL cholesterol (mmol/L) & $135.8 \pm 22.6$ & $137.4 \pm 20.3$ & 0.024 \\
\hline Glucose $(\mathrm{mg} / \mathrm{dl})$ & $85.3 \pm 19.4$ & $87.6 \pm 20.5$ & 0.058 \\
\hline \multicolumn{4}{|l|}{ Medications } \\
\hline ACE inhibitor or ARB (\%) & $63(30.1)$ & $67(31.9)$ & 0.466 \\
\hline Prednisone dose (mg/d) & $9.7(7.3-10.3)$ & $10.2(7.6-11.3)$ & 0.301 \\
\hline Calcineurin inhibitor (\%) & $135(64.5)$ & $129(61.4)$ & 0.061 \\
\hline Mycophenolate mofetil (\%) & $68(32.5)$ & $74(35.2)$ & 0.101 \\
\hline Sirolimus (\%) & $4(1.9)$ & $5(2.5)$ & 0.294 \\
\hline
\end{tabular}

Note: Data are presented as mean \pm SD for normally distributed data, as median (interquartile range) for nonnormally distributed data, and as $\mathrm{n}$ (\%) for categoric variables. Abbreviations: KT, kidney transplantation; IL-10, interleukin I0; BMI, body mass index; BP blood pressure; eGFR, estimated glomerular filtration rate; NT-proBNP, $\mathrm{N}$-terminal prohormone of B-type natriuretic peptide; HDL, high-density lipoprotein; LDL, low-density lipoprotein.

without a history of acute rejection, which implied that the association might be affected by acute rejection.

\section{Discussion}

Our study identified elevated serum IL-10 levels as an independent risk factor for cardiovascular mortality and all-cause mortality in KT patients. The significant association in regression models suggests that higher IL-10 levels may have value in predicting the risk of cardiovascular mortality and all-cause mortality in KT recipients.

IL-10 is an immunologic and inflammatory cytokine secreted mainly from activated macrophages, lymphocytes and monocytes. ${ }^{5}$ IL-10 can downregulate the inflammatory response by inhibiting all kinds of proinflammatory cytokines in various cells. ${ }^{6,7}$ IL-10 also has a cardiovascular protective effect and antiatherosclerotic properties in 


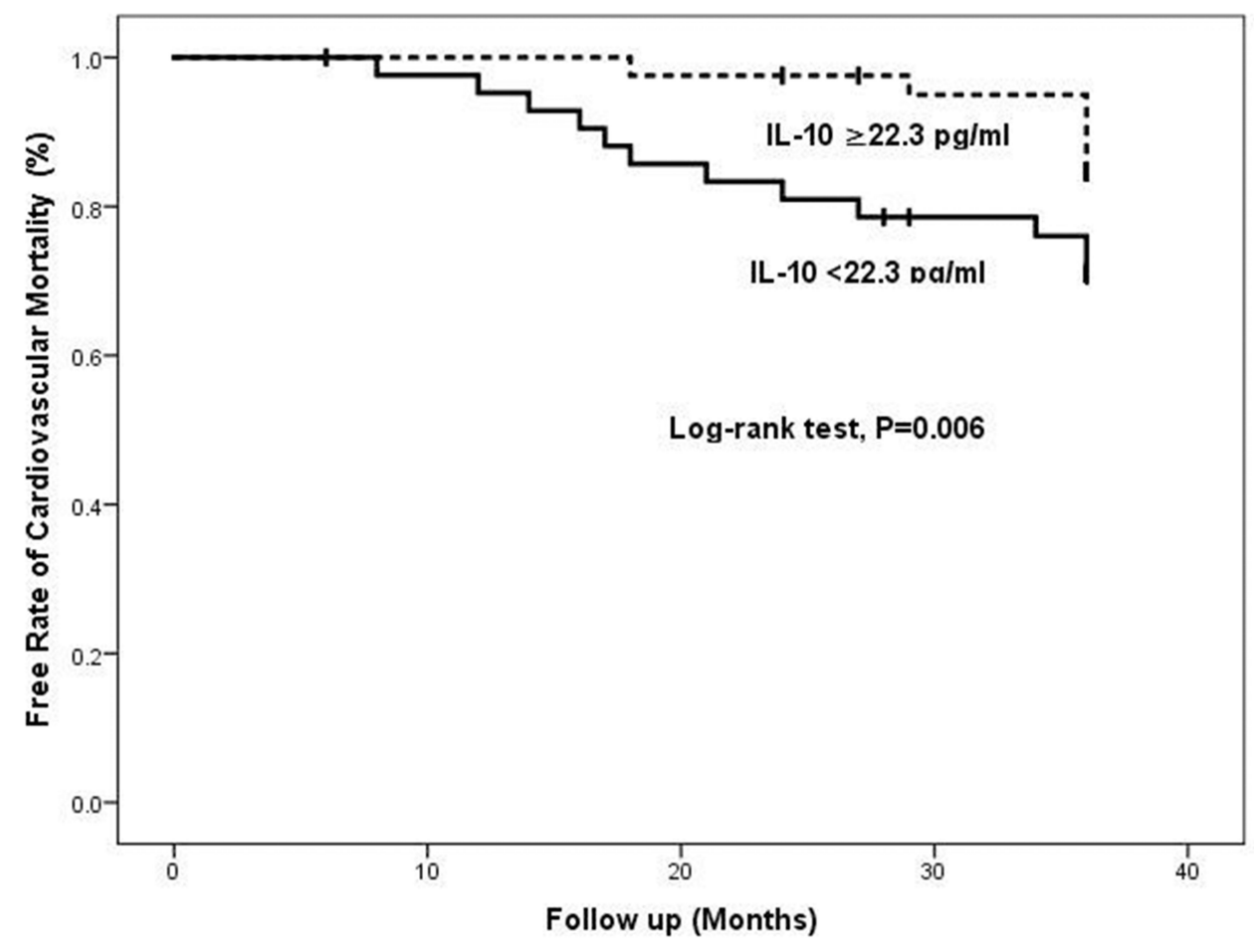

Figure I Kaplan-Meier analysis of cardiovascular mortality stratified into 2 groups by median level of serum IL-I0.

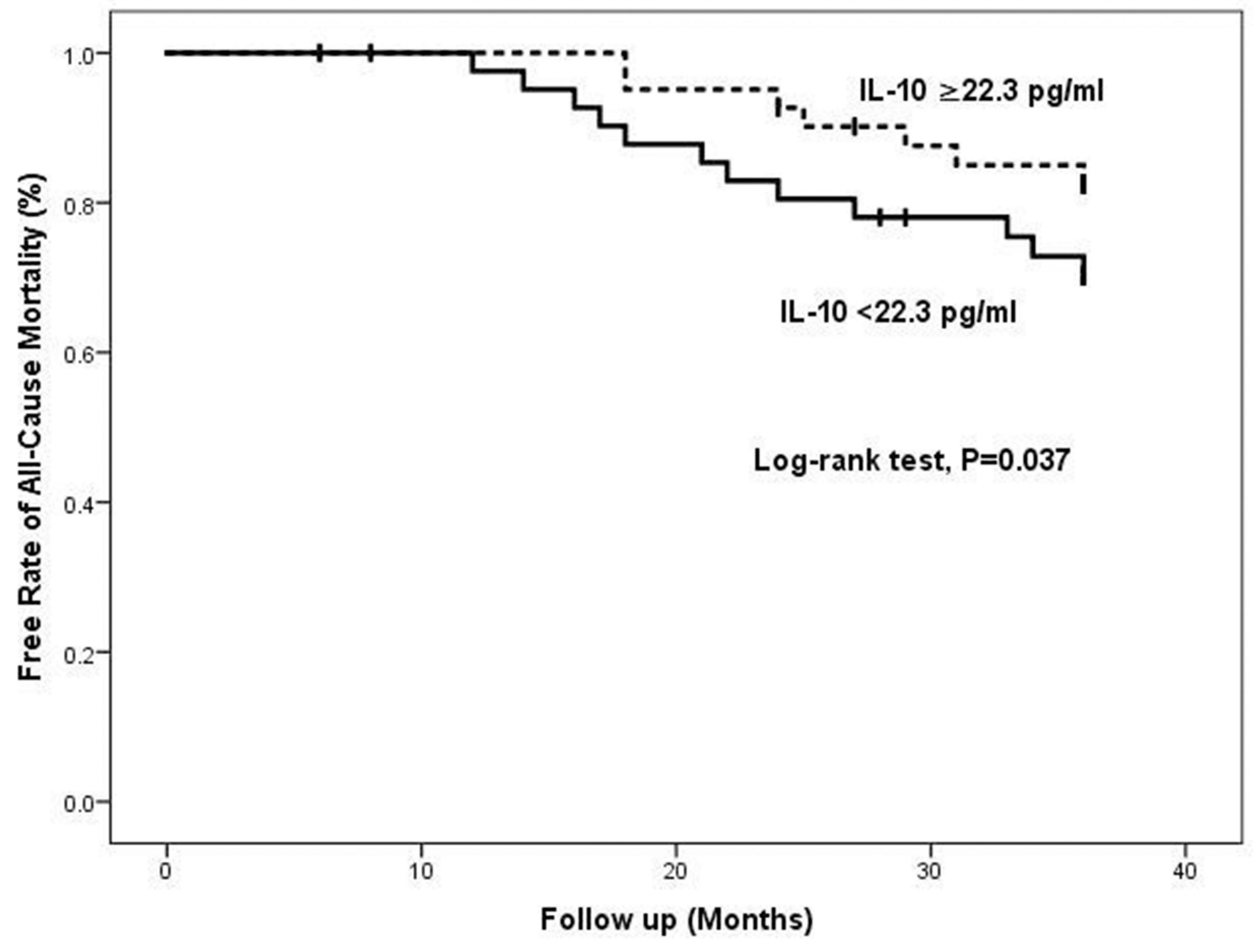

Figure 2 Kaplan-Meier analysis of all-cause mortality stratified into 2 groups by median level of serum IL-10.

addition to its broad anti-inflammatory activity. Previous studies suggested that IL-10 was found in atherosclerotic plaques, which may be a possible result of local secretion by tissue macrophages. ${ }^{7}$ IL-10 interferes with the process of atherosclerosis by reducing adhesion molecules, including intercellular adhesion molecule 1, CD60 L and 
Table 2 Multivariate Cox Regression Analysis of Predicting Cardiovascular Mortality in 419 Patients with KT

\begin{tabular}{|c|c|c|c|}
\hline $\begin{array}{l}\text { Serum IL-10 } \\
\text { Levels }\end{array}$ & Model I & Model 2 & Model 3 \\
\hline Quartile I (low) & 1.000 (ref.) & 1.000 (ref.) & 1.000 (ref.) \\
\hline Quartile 2 & $\begin{array}{c}1.21 \\
(1.11-1.65)\end{array}$ & $\begin{array}{c}1.15 \\
(1.08-1.61)\end{array}$ & $\begin{array}{c}1.13 \\
(1.04-1.57)\end{array}$ \\
\hline Quartile 3 & $\begin{array}{c}1.28 \\
(1.15-1.73)\end{array}$ & $\begin{array}{c}1.23 \\
(1.12-1.69)\end{array}$ & $\begin{array}{c}1.20 \\
(1.10-1.66)\end{array}$ \\
\hline Quartile 4 (high) & $\begin{array}{c}1.34 \\
(0.19-2.31)\end{array}$ & $\begin{array}{c}1.31 \\
(1.16-2.18)\end{array}$ & $\begin{array}{c}1.26 \\
(1.19-2.08)\end{array}$ \\
\hline$P$-trend & $<0.001$ & $<0.001$ & $<0.001$ \\
\hline
\end{tabular}

Notes: Model I: Adjusted for age and gender. Model 2: Adjusted for age, gender, BMI, current smoker, current drinker, cause of kidney disease, systolic and diastolic $\mathrm{BP}$ and laboratory indexes. Model 3: Adjusted for age, gender, BMI, current smoker, current drinker, cause of kidney disease, systolic and diastolic BP, laboratory indexes and medication.

Abbreviations: KT, kidney transplantation; IL-10, interleukin 10; BMI, body mass index; BP, blood pressure.

CD18. ${ }^{21,22}$ IL-10 also suppresses superoxide anion production and downregulates the production of matrix metalloproteinases, helping to stabilize atherosclerotic plaques. $^{22,23}$

Consistent with the antiatherosclerotic effects of IL-10, animal studies have also found that high expression of the IL-10 gene attenuates atherogenesis. ${ }^{24-26}$ However, some clinical studies have reported controversial results, namely that elevated serum levels of IL-10 are associated with

Table 3 Multivariate Cox Regression Analysis of Predicting AllCause Mortality in 419 Patients with KT

\begin{tabular}{|c|c|c|c|}
\hline $\begin{array}{l}\text { Serum IL- I } 0 \\
\text { Levels }\end{array}$ & Model I & Model 2 & Model 3 \\
\hline Quartile I (low) & I.000 (ref.) & I.000 (ref.) & I.000 (ref.) \\
\hline Quartile 2 & $\begin{array}{c}1.19 \\
(1.09-1.54)\end{array}$ & $\begin{array}{c}1.14 \\
(1.07-1.52)\end{array}$ & $\begin{array}{c}1.10 \\
(1.03-1.49)\end{array}$ \\
\hline Quartile 3 & $\begin{array}{c}1.25 \\
(1.13-1.68)\end{array}$ & $\begin{array}{c}1.23 \\
(1.10-1.63)\end{array}$ & $\begin{array}{c}1.21 \\
(1.07-1.57)\end{array}$ \\
\hline Quartile 4 (high) & $\begin{array}{c}1.31 \\
(1.15-1.99)\end{array}$ & $\begin{array}{c}1.28 \\
(1.12-1.90)\end{array}$ & $\begin{array}{c}1.25 \\
(1.11-1.82)\end{array}$ \\
\hline$P$-trend & 0.014 & 0.018 & 0.023 \\
\hline
\end{tabular}

Notes: Model I: Adjusted for age and gender. Model 2: Adjusted for age, gender, BMI, current smoker, current drinker, cause of kidney disease, systolic and diastolic BP and laboratory indexes. Model 3: Adjusted for age, gender, BMI, current smoker, current drinker, cause of kidney disease, systolic and diastolic BP, laboratory indexes and medication.

Abbreviations: KT, kidney transplantation; IL-10, interleukin 10; BMI, body mass index; BP, blood pressure.
Table 4 Sensitivity Analysis of Predicting Cardiovascular Mortality in 419 Patients with KT

\begin{tabular}{|c|c|c|c|}
\hline $\begin{array}{l}\text { Serum IL-I } 0 \\
\text { Levels }\end{array}$ & Model I & Model 2 & Model 3 \\
\hline Quartile I (low) & 1.000 (ref.) & I.000 (ref.) & I.000 (ref.) \\
\hline Quartile 2 & $\begin{array}{c}1.21 \\
(1.10-1.62)\end{array}$ & $\begin{array}{c}1.14 \\
(1.07-1.58)\end{array}$ & $\begin{array}{c}1.12 \\
(1.02-1.53)\end{array}$ \\
\hline Quartile 3 & $\begin{array}{c}1.27 \\
(1.14-1.72)\end{array}$ & $\begin{array}{c}1.22 \\
(1.11-1.65)\end{array}$ & $\begin{array}{c}1.18 \\
(1.07-1.61)\end{array}$ \\
\hline Quartile 4 (high) & $\begin{array}{c}1.32 \\
(1.15-2.24)\end{array}$ & $\begin{array}{c}1.27 \\
(1.14-2.03)\end{array}$ & $\begin{array}{c}1.24 \\
(1.12-1.98)\end{array}$ \\
\hline$P$-trend & $<0.001$ & $<0.001$ & $<0.001$ \\
\hline
\end{tabular}

Notes: Model I: Adjusted for age, gender and dialysis duration. Model 2: Adjusted for age, gender, BMI, current smoker, current drinker, cause of kidney disease, systolic and diastolic BP, laboratory indexes and dialysis duration. Model 3: Adjusted for age, gender, BMI, current smoker, current drinker, cause of kidney disease, systolic and diastolic BP, laboratory indexes, medication and dialysis duration.

Abbreviations: KT, kidney transplantation; IL-10, interleukin 10; BMI, body mass index; BP, blood pressure.

poor clinical outcomes in patients with acute coronary syndrome. ${ }^{7}$ In contrast, another study found that higher IL-10 levels at baseline can predict a higher risk of cardiovascular events in patients with acute myocardial infarction. ${ }^{8}$

Some hypotheses may explain the discrepancies in these previous studies. First, similar to other inflammatory factors, the levels of serum IL-10 can change rapidly over

Table 5 Sensitivity Analysis of Predicting All-Cause Mortality in 419 Patients with KT

\begin{tabular}{|l|c|c|c|}
\hline $\begin{array}{l}\text { Serum IL-I0 } \\
\text { Levels }\end{array}$ & Model I & Model 2 & Model 3 \\
\hline Quartile I (low) & $\mathrm{I} .000$ (ref.) & $\mathrm{I} .000$ (ref.) & $\mathrm{I} .000$ (ref.) \\
\hline Quartile 2 & $\begin{array}{c}\mathrm{I} . \mathrm{I} 7 \\
(\mathrm{I} .07-\mathrm{I} .52)\end{array}$ & $\begin{array}{c}\mathrm{I} . \mathrm{I3} \\
(\mathrm{I} .05-\mathrm{I} .50)\end{array}$ & $\begin{array}{c}\mathrm{I} . \mathrm{II} \\
(\mathrm{I} .0 \mathrm{I}-\mathrm{I} .47)\end{array}$ \\
\hline Quartile 3 & $\begin{array}{c}\mathrm{I} .22 \\
(\mathrm{I} I \mathrm{I}-\mathrm{I} .64)\end{array}$ & $\begin{array}{c}\mathrm{I} .20 \\
(\mathrm{I} .09-\mathrm{I} .6 \mathrm{I})\end{array}$ & $\begin{array}{c}\mathrm{I} .19 \\
(\mathrm{I} .06-\mathrm{I} .55)\end{array}$ \\
\hline Quartile 4 (high) & $\begin{array}{c}\mathrm{I} .29 \\
(\mathrm{I} .14-\mathrm{I} .95)\end{array}$ & $\begin{array}{c}\mathrm{I} .27 \\
(\mathrm{I} . \mathrm{I}-\mathrm{I} .92)\end{array}$ & $\begin{array}{c}\mathrm{I} .22 \\
(\mathrm{I} .09-\mathrm{I} .87)\end{array}$ \\
\hline P-trend & 0.015 & 0.020 & 0.025 \\
\hline
\end{tabular}

Notes: Model I: Adjusted for age, gender and dialysis duration. Model 2: Adjusted for age, gender, BMl, current smoker, current drinker, cause of kidney disease, systolic and diastolic BP, laboratory indexes and dialysis duration. Model 3: Adjusted for age, gender, BMl, current smoker, current drinker, cause of kidney disease, systolic and diastolic BP, laboratory indexes, medication and dialysis duration.

Abbreviations: KT, kidney transplantation; IL-10, interleukin I0; BMI, body mass index; BP, blood pressure. 
Table 6 Stratified Analysis of Predicting Cardiovascular Mortality and All-Cause Mortality in 4I9 Patients with KT

\begin{tabular}{|l|c|c|c|}
\hline Serum IL-I0 Levels & Model I & Model 2 & Model 3 \\
\hline Cardiovascular mortality & & & $1.40(1.16-2.85)$ \\
History of acute rejection (Yes) & $1.46(1.18-2.82)$ & $1.42(1.17-2.79)$ & 0.026 \\
P value & 0.012 & 0.014 & $1.05(0.92-1.84)$ \\
History of acute rejection (No) & $1.13(1.02-2.3)$ & $1.09(0.96-1.99)$ & 0.181 \\
P value & 0.37 & 0.53 & $1.34(1.13-2.67)$ \\
\hline All-cause mortality & & $1.36(1.15-2.7 I)$ & 0.031 \\
History of acute rejection (Yes) & $1.39(1.17-2.75)$ & 0.017 & $1.01(0.87-1.79)$ \\
P value & 0.013 & $1.02(0.89-1.81)$ & 0.214 \\
History of acute rejection (No) & $1.04(0.91-1.83)$ & 0.201 & \\
P value & 0.189 & & \\
\hline
\end{tabular}

Note: Adjusted for age, gender, BMI, current smoker, current drinker, cause of kidney disease, systolic and diastolic BP, laboratory indexes and medication. Abbreviations: KT, kidney transplantation; IL-10, interleukin I0; BMI, body mass index; BP, blood pressure.

time, and the time of sampling may affect the results. Most studies only collected one blood sample. Second, IL-10 may have some harmful effects, and elevated IL-10 levels may reflect a counterregulatory mechanism or compensation in response to a heightened inflammatory state. ${ }^{27}$ Third, the survival benefit of IL-10 may require a longer follow-up time. Variable follow-up durations may have affected the observed results in these previous studies. Fourth, circulating IL-10 levels do not accurately reflect the levels of IL-10 in tissues, especially in atherosclerotic plaques. $^{28}$

To solve this controversy, one study followed patients with ACS for 5 years and found that increased serum levels of IL-10 at baseline are a strongly significant predictor for adverse cardiovascular outcomes, which is consistent with our results and is also consistent with previous evidence that high IL-10 levels are associated with an increased rate of cardiovascular events in patients with $\mathrm{CKD} .{ }^{29}$ Moreover, IL-10 levels have also been found to be linked to a higher risk of cardiovascular events in the general population. ${ }^{30}$ Although KT patients in the higher IL-10 tertile tend to have a high cardiovascular risk, the associations of serum IL-10 with cardiovascular mortality and all-cause mortality remained independent and significant after adjusting for related confounding factors, which suggests that IL-10 is a strong and independent risk factor in KT patients.

In summary, similar to other inflammatory markers, serum levels of IL-10 were significantly higher in patients with a lower estimated glomerular filtration rate (eGFR). This seemingly paradoxical increase in an antiatherosclerotic cytokine and its independent association with adverse cardiovascular outcomes might be explained by a compensatory increase in response to a chronic inflammatory state in patients with KT.

Our study has some limitations. Although some confounders were taken into account, including renal function, which may be a very important determinant of IL-10 levels, the presence of other confounders cannot be fully excluded because many determinants of IL-10 are currently unknown. The sensitivity analyses in KT patients with eGFR as a covariate reduced the risk of confounding by abnormal renal function. Hypertensive and diabetic nephropathy were not representative in this study, limiting the generalizability of our results because our cohort consisted of an all Asian population with KT. We could not perform a repeated measures analysis in this cohort due to the measurement of IL-10 at only a single time point, which may introduce some bias. A Cox regression model using a relatively large number of covariates might cause overfitting of the model. Finally, we did not have enough cardiac data from echocardiography or biochemical parameters to investigate the underlying mechanisms of the independent association between IL-10 and cardiovascular mortality and all-cause mortality. Since this is a prospective longitudinal study, it cannot establish causality in the association between serum IL-10 levels and cardiovascular mortality after KT. Additionally, some data concerning cardiovascular mortality and all-cause mortality were collected by telephone calls from the patients or treating physician and not from formal death certificate or medical records.

\section{Conclusions}

In conclusion, we provided the first evidence that serum IL10 may be considered an independent predictor of 
cardiovascular mortality and all-cause mortality after KT. The results of our study showed that elevated levels of serum IL-10 might be independently associated with cardiovascular outcomes. We hope our results will encourage the conduction of prospective studies to better clarify the role of serum IL-10 in the development of cardiovascular mortality and all-cause mortality.

\section{Funding}

There is no funding to report.

\section{Disclosure}

The authors report no conflicts of interest in this work.

\section{References}

1. Parsikia A, Ortiz C, Ortiz J, et al. Re: hepatitis C virus infection in end-stage renal disease and kidney transplantation. Transplant Int. 2015;28(5). doi:10.1111/tri.12492

2. Jardine AG, Gaston RS, Fellstrom BC, et al. Prevention of cardiovascular disease in adult recipients of kidney transplants. Lancet. 2011;378(9800):1419-1427. doi:10.1016/S0140-6736(11)61334-2

3. Sam WJ, Chamberlain CE, Lee SJ, et al. Associations of ABCB1 and IL-10 genetic polymorphisms with sirolimus-induced dyslipidemia in renal transplant recipients. Transplantation. 2011;94(9):971-977. doi:10.1097/TP.0b013e31826b55e2

4. Serrano OK, Bangdiwala A, Vock D, et al. Risk factors for developing adult cardiovascular disease in children who received a kidney transplant: analysis of 1055 kidney transplants between 1963-2015 at a single institution. J Am Coll Surg. 2016;223(4):S148-S149. doi:10.1016/j.jamcollsurg.2016.06.319

5. de Vries JE. Immunosuppressive and Anti-inflammatory properties of interleukin 10. Ann Med. 1995;27(5):537-541. doi:10.3109/ 07853899509002465

6. Morita Y, Yamamura M, Kashihara N, et al. Increased production of interleukin-10 and inflammatory cytokines in blood monocytes of hemodialysis patients. Res Commun Mol Pathol Pharmacol. 1997;98(1):19-33.

7. Stenvinkel P, Ketteler M, Johnson RJ; Publications S. IL-10, IL-6, and TNF-alpha: central factors in the altered cytokine network of uremia-the good, the bad, and the ugly. Kidney Int. 2005;67 (4):1216-1233. doi:10.1111/j.1523-1755.2005.00200.x

8. Maelarstig A, Eriksson P, Hamsten A, et al. Raised interleukin-10 is an indicator of poor outcome and enhanced systemic inflammation in patients with acute coronary syndrome. Heart. 2008;94(6):724-729. doi:10.1136/hrt.2007.119271

9. Girndt M, Kaul H, Sester U, et al. Anti-inflammatory interleukin-10 genotype protects dialysis patients from cardiovascular events. Kidney Int. 2002;62(3):949-955. doi:10.1046/j.1523-1755.2002.00504.x

10. Straczkowski M, Kowalska I, Nikolajuk A, et al. Plasma interleukin-10 concentration is positively related to insulin sensitivity in young healthy individuals. Diabetes Care. 2005;28(8):2036-2037. doi:10.2337/diacare.28.8.2036

11. Jose I, Jumil LK, Couto PJ, et al. Immune reconstitution inflammatory syndrome occurring in a kidney transplant patient with extrapulmonary tuberculosis. Case Rep Transplant. 2017;2017:1-5.

12. LópezGómez JM, Pérez-Flores I, Jofré R, et al. Presence of a failed kidney transplant in patients who are on hemodialysis is associated with chronic inflammatory state and erythropoietin resistance. $J \mathrm{Am}$ Soc Nephrol. 2004;15(9):2494. doi:10.1097/01.ASN.0000137879. 97445.6E
13. Agarwal R. Proinflammatory effects of oxidative stress in chronic kidney disease: role of additional angiotensin II blockade. $\mathrm{Am}$ J Physiol Renal Physiol. 2003;284(4):F863-9. doi:10.1152/ ajprenal.00385.2002

14. Ortega $\mathrm{O}$, Cobo G, Rodríguez I, et al. Lower plasma sodium is associated with a microinflammatory state among patients with advanced chronic kidney disease. Nephron. 2014;128(3-4):312-318. doi:10.1159/000368116

15. Yokoe T, Minoguchi K, Matsuo H. Elevated levels of C-reactive protein and interleukin- 6 in patients with obstructive sleep apnea syndrome are decreased by nasal continuous positive airway pressure. Circulation. 2003;107(8):1129-1134. doi:10.1161/01.CIR.0000052627.99976.18

16. Naito K, Ikoma F, Thüroff JW, et al. C-reactive protein and cardiovascular diseases. Int J Angiol. 2003;12(01):1-12. doi:10.1007/s00547-003-1018-y

17. Lin $\mathrm{YH}$, Glei $\mathrm{D}$, Weinstein $\mathrm{M}$, et al. Additive value of interleukin-6 and C-reactive protein in risk prediction for all-cause and cardiovascular mortality among a representative adult cohort in Taiwan. J Formos Med Assoc. 2017:S0929664617300967.

18. Langenberg C, Bergstrom J, Scheidt-Nave C, et al. Cardiovascular death and the metabolic syndrome: role of adiposity-signaling hormones and inflammatory markers. Diabetes Care. 2006;29 (6):1363-1369. doi:10.2337/dc05-2385

19. Song S, Ling-Hu H, Roebuck KA, et al. Interleukin-10 inhibits interferon- $\gamma$-induced intercellular adhesion molecule-1 gene transcription in human monocytes. Blood. 1997;89(12):4461-4469. doi:10.1182/blood.V89.12.4461

20. Levey AS, Becker C, Inker LA, et al. Glomerular filtration rate and albuminuria for detection and staging of acute and chronic kidney disease in adults: a systematic review. JAMA. 2015;313(8):837. doi:10.1001/jama.2015.0602

21. von der Thüsen JH, Kuiper J, Fekkes ML, et al. Attenuation of atherogenesis by systemic and local adenovirus-mediated gene transfer of interleukin-10 in LDLr-/- mice. FASEB J. 2001;15 (14):2730-2732. doi:10.1096/fj.01-0483fje

22. Mallat Z, Heymes C, Ohan J, et al. Expression of interleukin-10 in advanced human atherosclerotic plaques: relation to inducible nitric oxide synthase expression and cell death. Arterioscler Thromb Vasc Biol. 1999;19(3):611-616. doi:10.1161/01.ATV.19.3.611

23. Pinderski Oslund LJ, Hedrick CC, Olvera T, et al. Interleukin-10 blocks atherosclerotic events in vitro and in vivo. Arterioscler Thromb Vasc Biol. 1999;19(12):2847-2853. doi:10.1161/01.ATV.19.12.2847

24. Cavusoglu E, Marmur JD, Hojjati MR, et al. Plasma interleukin-10 levels and adverse outcomes in acute coronary syndrome. Am J Med. 2011;124(8):724-730. doi:10.1016/j.amjmed.2011.02.040

25. Eskdale J, Gallagher G. A polymorphic dinucleotide repeat in the human IL-10 promoter. Immunogenetics. 1995;42(5):444. doi:10.1007/BF00179416

26. Macdermott RP, Nash GS, Bertovich MJ, et al. Alterations of IgM, $\mathrm{IgG}$, and IgA Synthesis and secretion by peripheral blood and intestinal mononuclear cells from patients with ulcerative colitis and Crohn's disease. Gastroenterology. 1981;81(5):844-852. doi:10.1016/S0016-5085(81)80107-2

27. Koch W, Kastrati A, Böttiger C, et al. Interleukin-10 and tumor necrosis factor gene polymorphisms and risk of coronary artery disease and myocardial infarction. Atherosclerosis. 2001;159 (1):137-144. doi:10.1016/S0021-9150(01)00467-1

28. Brunet P, Capo C, Dellacasagrande J, et al. IL-10 synthesis and secretion by peripheral blood mononuclear cells in haemodialysis patients. Nephrol Dial Transplant. 1998;13(7):1745-1751.

29. Woowiec D, Frydecka I, Kosmaczewska A, et al. Interleukin-4 and interleukin-10 secretion by peripheral blood $\mathrm{T}$ cells in patients with chronic B-cell leukemia. Adv Clin Exp Med. 2003;12(1):45-49.

30. Taniguchi T, Koido Y, Aiboshi J, et al. Change in the ratio of interleukin-6 to interleukin-10 predicts a poor outcome in patients with systemic inflammatory response syndrome. Crit Care Med. 1999;27(7):1262-1264. doi:10.1097/00003246-199907000-00005 


\section{Publish your work in this journal}

Risk Management and Healthcare Policy is an international, peerreviewed, open access journal focusing on all aspects of public health, policy, and preventative measures to promote good health and improve morbidity and mortality in the population. The journal welcomes submitted papers covering original research, basic science, clinical \& epidemiological studies, reviews and evaluations, guidelines, expert opinion and commentary, case reports and extended reports. The manuscript management system is completely online and includes a very quick and fair peer-review system, which is all easy to use. Visit http://www.dovepress.com/testimonials.php to read real quotes from published authors.

Submit your manuscript here: https://www.dovepress.com/risk-management-and-healthcare-policy-journal 